\title{
VARIABLE STARS IN DWARF SPHEROIDAL GALAXIES
}

\author{
STEVEN VAN AGT \\ University of Nijmegen, Nijmegen, The Netherlands
}

\section{Introduction}

Interest in dwarf spheroidal galaxies is motivated by a number of reasons; an important one on the occasion of this colloquium is the abundance of variable stars. The theory of stellar evolution and stellar pulsations is now able to predict from theoretical considerations characteristic properties of variable stars in the colour-magnitude diagram (Iben, 1971). By observing the variable stars in the field, and in as wide a selection of objects as possible, more insight can be obtained into the history of the oldest members of our Galaxy (the globular clusters) and of the dwarf spheroidal galaxies in the Local Group. It is worthwhile to explore the spheroidal galaxies as observational tests for the theoretical predictions of conditions in space away from our Galaxy. The numbers of variable stars in the dwarf spheroidal galaxies are such that we may expect well-defined relations to emerge once reliable magnitude sequences have been set up, the variable stars found, and their periods determined. Six dwarf spheroidal galaxies are presently known in the Local Group within a distance of $250 \mathrm{kpc}$. In Table I, which lists members of the Local Group, they are at the lowluminosity end of the sequence of elliptical galaxies (van den Bergh, 1968).

How complete this number of spheroidals in the Local Group may be is uncertain. The discoveries are strongly affected by selection.

TABLE I

Local group members

\begin{tabular}{lll}
\hline Name & Type & $M_{v}$ \\
\hline M31 = NGC 224 & Sb I-II & -21.1 \\
Galaxy & Sb or Sc & -20 \\
M33 = NGC 598 & Sc II-III & -18.9 \\
LMC & Ir or SBc III-IV & -18.5 \\
SMC & Ir IV or Ir IV-V & -16.8 \\
NGC 205 & E6p & -16.4 \\
M32= NGC 221 & E2 & -16.4 \\
NGC 6822 & Ir IV-V & -15.7 \\
NGC 185 & dE0 & -15.2 \\
NGC 147 & dE4 & -14.9 \\
IC 1613 & Ir V & -14.8 \\
Fornax & Spheroidal & -13.6 \\
Sculptor & Spheroidal & -11.7 \\
Leo I & Spheroidal & -11.0 \\
Leo II & Spheroidal & -9.4 \\
Ursa Minor & Spheroidal & -8.8 \\
Draco & Spheroidal & -8.6 \\
\hline
\end{tabular}


The Sculptor dwarf galaxy was discovered quite accidentally by Shapley (1938a, b), and the Fornax system in a search of the southern polar-cap region on existing plates at Harvard Observatory. However the limiting magnitudes of these plates is only a little fainter than 18 th magnitude and the plate scale is large. The Sculptor and Fornax dwarf galaxies are the only ones discovered in the southern sky. The northern sky has been searched systematically by Wilson (1955) on the plates of the Palomar Sky Survey.

The lack of a survey of the southern sky comparable in limiting magnitude and angular resolution to the Palomar Sky Survey greatly contributes to the uncertainties in the completeness. To find the extended Ursa Minor system even on the Sky Survey plates is difficult at galactic latitude $b=+45^{\circ}$ due to the low stellar surface density in this galaxy. A ten times higher surface density in the field closer to the galactic plane would allow this spheroidal to completely escape detection.

The apparent lack of spheroidal galaxies at galactic latitudes smaller than $b=35^{\circ}$ is better explained as observational selection near our Galaxy than as another example of the apparent preference of dwarf galaxies to avoid low local galactic latitudes near large spirals (Holmberg, 1969). The recognition on the photographic emulsion of a dwarf spheroidal galaxy at large distances from the Galaxy is difficult (Reaves, 1956). Intrinsically faint galaxies have their light less concentrated towards the center and are therefore less easily recorded. The use of newly developed emulsions can prove rewarding, as is indicated by the recent discovery of three new dwarf spheroidal galaxies near M31 by van den Bergh (1972). The Kodak IIIaJ emulsion on which these dwarf spheroidals were discovered has an extremely high linear resolution and is especially suitable for recording faint magnitudes (Marchant and Millikan, 1965).

From dynamical considerations Hodge and Michie (1969) find it unlikely that the dwarf spheroidal galaxies ever were very close to the Galaxy, since close encounters would have completely disrupted the spheroidal, which is unimpressive in terms of mass : $10^{5}$ to $5 \times 10^{6} \mathfrak{M}_{\odot}$.

Estimates of the total number of dwarf spheroidal galaxies in the Local Group vary from 8 (Wilson, 1955) to more than 192 (van den Bergh, 1968), if one wishes to assume that spheroidal galaxies are homogeneously distributed out to $400 \mathrm{kpc}$ in the Local Group.

Table II gives the positions in galactic coordinates of the nearby spheroidal galaxies.

TABLE II

Coordinates of nearby spheroidal galaxies

\begin{tabular}{lll}
\hline Name & $l$ & $b$ \\
\hline Fornax & $237^{\circ}$ & $-66^{\circ}$ \\
Sculptor & 286 & -83 \\
Leo I & 226 & +49 \\
Leo II & 219 & +67 \\
Ursa Minor & 103 & +45 \\
Draco & 86 & +35 \\
\hline
\end{tabular}




\section{Variable Stars}

For a full survey of the distribution of variable stars in extended dwarf spheroidal galaxies, wide-field telescopes can be used efficiently to avoid the numerous exposures off-set from the center of the galaxy necessary if telescopes of small angular field are used. The brightest stars in the nearest of the intrinsically faint dwarf galaxies reach approximately $B=17 \mathrm{mag}$. The surface density of the stars down to the luminosity of the horizontal branch remains generally small, and for the central regions is 10 to 100 times higher than in the surrounding field. Telescopes with moderate angular resolution resolve the central regions well enough to allow inspection of the individual stars, and thus permit the search for and photometry of variable stars.

The first detailed investigation of individual stars in dwarf spheroidal galaxies was made by Baade and Hubble (1939) in the Sculptor galaxy. The authors reported 40 variable stars in the central region, but periods could not be derived from the limited number of 100-in. Mount Wilson plates. The range in stellar brightness and the distribution of the observations in time led Baade to the conclusion that the new variable stars were most likely RR Lyrae, with the exception of two variable stars with Cepheid characteristics.

Further investigation of the Sculptor and Fornax systems was prevented at that time by the lack of instrumental facilities in the south. In 1948 the 74-in. Radcliffe telescope became operational and new observations of the Sculptor galaxy were obtained by Thackeray. He discovered (Thackeray, 1950a) more than 230 variable stars in Sculptor. His material is well suited for deriving periods and is presently at the Dept. of Astronomy of the University of Nijmegen, the Netherlands, for further reduction.

After the discovery of the four spheroidal dwarf galaxies in the northern sky by Wilson (1955), Baade obtained large numbers of 200-in. plates of the dwarf spheroidal galaxies in Draco, Leo, Ursa Minor, and also of NGC 147 and NGC 185. His observations aimed at finding the variable stars now known to be present, and probably in large numbers, in the central regions of these objects, and at deriving their periods and magnitudes. For the Draco system the reductions are extensively discussed by Baade and Swope (1961). The Leo II dwarf galaxy is discussed in a progress report by Swope (1967) and the results for the Ursa Minor system by van Agt (1967).

New observations of the Sculptor system have been obtained recently by observers from the David Dunlap Observatory. These observations were made with the 24/36in. Michigan Curtiss Schmidt telescope at Cerro Tololo, Chile. The $5^{\circ} \times 5^{\circ}$ field of the Curtiss Schmidt telescope, which has a plate scale of $97.2 \mathrm{~mm}^{-1}$, accommodates the dimensions of the Sculptor system well.

Intercomparison of ten plate pairs of the Sculptor system yielded a rich harvest of over 500 variable stars. The total number of variable stars in this spheroidal galaxy, including those discovered earlier by Baade and Hubble, Thackeray and collaborators, Helen Hogg, who also blinked a plate pair, and the author is now over 600 . The expected total number of variable stars is well over 850 as estimated from 
an application of van Gent's method (van Gent, 1933) in elliptical zones around the center and with constant stellar surface density. Discovery chances vary with distance from the center due to the decreasing surface density of the stars. In estimating the expected total number of variable stars, varying discovery chances with amplitude of the variable have not been taken into account. It is expected that discoveries of the $c$-type variables are strongly affected by selection.

The Fornax system is now under investigation by Demers at Laurentian University, Sudbury, Ontario. Demers obtained his photographic observations of the Fornax dwarf galaxy with the Curtiss Schmidt at Cerro Tololo. A number of plates have been measured with the GALAXY machine in Edinburgh, Scotland, and it is expected that variable stars can be picked out from the magnitude of the standard deviations determined for all the stars which are measured with the GALAXY machine (Demers, 1972).

The Leo I dwarf galaxy is being investigated by Hodge. The nearness of the bright star Regulus makes observing photographically difficult due to reflections in the optical system of the telescope. Recent observations yielded a number of short period Cepheids (Hodge, in preparation).

The absolute number of variable stars which has been discovered in dwarf spheroidal galaxies so far is large compared to the numbers of variable stars in galactic globular clusters. Only NGC 5272 (M3), NGC 5139 ( $\omega$ Cen), IC 4499 and NGC 5904 (M5), with more than one hundred variable stars each, are comparable.

The present state of the investigations is summarized in Table III.

TABLE III

Variables in spheroidal galaxies

\begin{tabular}{lcl}
\hline Name & $\begin{array}{c}\text { Total No. } \\
\text { variables } \\
\text { discovered }\end{array}$ & Remarks \\
\hline $\begin{array}{l}\text { Fornax } \\
\text { Draco }\end{array}$ & - & in progress (Demers, 1972) \\
Ursa Minor & 261 & Baade and Swope (1961) \\
Sculptor & 92 & van Agt (1967) \\
Leo I & 603 & in progress (van Agt) \\
Leo II & - & in progress (Hodge) \\
\hline
\end{tabular}

In absolute numbers the Sculptor system is very rich in variable stars. By comparing the luminosity functions of NGC 5272 (Sandage, 1954) and the Sculptor system (Hodge, 1965) we derive that per giant star in a luminosity interval of 0.8 mag. near the top of the giant branch the Sculptor system may contain 1.5 times the number of variable stars in the globular cluster.

Variable stars have been traced in the Sculptor system out to distances of well over $60^{\prime}$. The limiting radius determined by Hodge (1961) from starcounts on a number of plates is $53^{\prime}$. The scaled surface density of the variable stars does not agree well with the surface density curve derived from the starcounts. 
New starcounts have been obtained by Annelies van Agt (1972, unpublished) on the same plates as used for finding the variable stars. The observations are processed with an electronic computer and the results are plotted as isodensity contours. The new contours indicate that the outer variables might well belong to the Sculptor dwarf galaxy. To determine whether the variable stars in the Sculptor system are arranged in a wider distribution than the stars in the giant branch, needs a more extensive reduction of the new observations. So far, only for the Sculptor system have the variable stars been traced over the full extent of the system. It has been difficult to obtain reliable standard sequences to sufficiently faint limiting magnitudes to calibrate the brightnesses of the stars in the spheroidal systems. Photoelectric sequences have been obtained for the Draco dwarf galaxy and for the Leo II system (Baade and Swope, 1961; Swope, 1967). The photoelectric sequence set up by Hodge (1965) for the Sculptor system does not reach faint enough to calibrate the RR Lyrae stars. Generally photographic transfers have been used to calibrate the stars in the spheroidal galaxies. For the Leo II system observations with the Racine prism have also been obtained. New calibrating techniques are now used to extend the standard sequences from relatively easy to obtain photoelectric measures of bright stars towards fainter limiting magnitudes, specifically with the electronographic camera and the Racine prism.

The magnitudes derived by Walker (1970) for the globular cluster Kron 3 in the Small Magellanic Cloud have been used for a photographic transfer to the Sculptor dwarf galaxy beyond the apparent magnitudes of the RR Lyrae stars in an effort to determine the magnitude scale somewhat more accurately. But much more attention should be given to determining correct magnitude scales in the dwarf spheroidal galaxies without the use of photographic transfers. Period-colour and period-amplitude relations can be seriously distorted by incorrect magnitude sequences. Table IV summarizes the present situation regarding the calibration of the magnitudes in the spheroidal galaxies.

The magnitudes given in the table are the limiting magnitudes.

Table $\mathrm{V}$ lists the variable stars in some dwarf spheroidal galaxies in more detail.

TABLE IV

Magnitude calibrations in spheroidal galaxies

\begin{tabular}{lccll}
\hline Name & $\begin{array}{l}\text { Photoelectric } \\
\text { B }\end{array}$ & $V$ & $\begin{array}{l}\text { Photographic } \\
B\end{array}$ & $V$ \\
\hline Fornax & & & $20.0(1)$ & \\
Draco & $21.3(2)$ & $20.5(2)$ & $\begin{array}{l}21.8(2) \\
21.6(3)\end{array}$ & $21.1(2)$ \\
Ursa Minor & & & $(4)$ & \\
Leo I & $(4)$ & & $22.0(5)$ & $20.8(5)$ \\
Leo II & & & $21.5(7)$ & \\
Sculptor & $19.8(6)$ & $18.6(6)$ & & \\
\hline
\end{tabular}

Remarks: (1) Demers (1972); (2) Baade and Swope (1961); (3) van Agt, (1967); (4) Hodge (unpublished); (5) Swope (1967); (6) Hodge (1965); (7) van Agt (unpublished). 


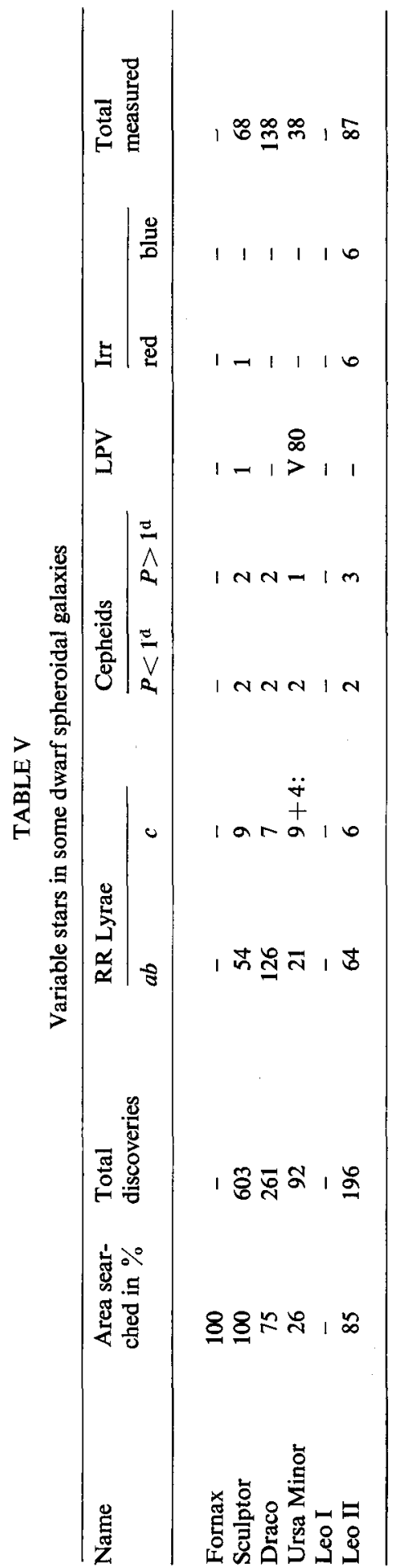


The reduction of the Fornax plates by Demers (1972) are not yet complete.

In the Sculptor dwarf spheroidal galaxy periods have been determined for a limited number of variable stars by Thackeray (1950b) and by van Agt (in preparation). Of the 63 variable stars with known periods $16 \%$ are $c$-type variables. This preliminary result indicates that in the Sculptor system $c$-type variables are probably not as scarce as in the Draco system.

In the field of Sculptor there are four short period variables with periods near one day. Included among these four is one variable with a period near 0.5 and approximately 0.5 mag. brighter than the RR Lyrae stars in this dwarf galaxy.

Variable stars with periods of less than one day to over one day, but not exceeding 3.0, and brighter than the other RR Lyrae stars in the dwarf spheroidal, are also in the Ursa Minor system (van Agt, 1967) and the Leo II system (Swope, 1967) and were discovered recently by Hodge in Leo I.

At a distance of $14^{\prime}$ north of the centre of the Sculptor dwarf galaxy a long period variable star has been found on the Curtiss Schmidt plates. From the 74-in. plates we find that the time of rise to maximum and of decline to minimum is of the order of 120 days. A definite period has not been derived but observations over a long time interval indicate that a period near 150 days is possible.

The amplitude of the long period variable derived from plates with a IIIaJ emulsion exposed through a GG13 filter is $2.5 \mathrm{mag}$. The $B_{j}$ magnitude is related to the $B V$ system through the equation $B_{j}=B-0.10(B-V)$. The $B_{j}$ luminosity at maximum light is comparable to the brightest giant branch stars. The colour-magnitude diagram as derived by Hodge (1965) has a giant branch extending to large $(B-V)$ values. At least one of the very red stars at the tip of the giant branch is a variable star with small amplitude and probably irregular period.

The Ursa Minor system is the least rich in variable stars and also appears on the sky as the least well-defined. The $c$-type variables are relatively frequent in this Galaxy, forming $35 \%$ of the RR Lyrae population in the central region.

For one variable in the Ursa Minor system, $\mathrm{V} \mathrm{80,} \mathrm{the} \mathrm{period} \mathrm{is} \mathrm{uncertain} \mathrm{and} \mathrm{clas-}$ sifying this variable as long period might be erroneous. The mean luminosity in $B$ is about 0.5 mag. brighter than the RR Lyrae stars. A period close to one day is not excluded.

The Ursa Minor system contains among the bright short period variable stars two variables with periods well below one day and with a luminosity about $0.5 \mathrm{mag}$. brighter than the RR Lyrae stars.

The Draco system contains more than 260 variable stars (Swope, 1961) within a radius of $24^{\prime}$ from the centre of the system, which has (Hodge, 1964) a limiting radius of $26^{\prime}$. The discoveries in the Draco system are complete within 6.3 from the centre and down to amplitudes in $B$ of 0.4 mag. Only the stars in the central region have been measured. In the Draco system no long period or irregular variable stars have been discovered, although the distribution of the plates in time certainly would allow this.

The giant branch of the Draco system does not reach the very red colours observed in the colour-magnitude diagram of the Sculptor system. 
At $B-V=1.4$ the giant branch is approximately 3.0 mag. brighter than the RR Lyrae stars. The giant branch is possibly double, but the number of stars in this region is small. From the luminosity of the giant branch it is expected that the stars in the Draco system are very metal poor. The very strong concentration of stars on the horizontal branch towards the red does not contradict this result since the distribution of the stars over the horizontal branch is not uniquely related to metallicity (Iben, 1971).

Variable stars brighter than the RR Lyrae stars have also been discovered in the Draco system.

The variable V 203, which has a small amplitude and is almost constant in luminosity during one observing season, falls above the horizontal branch in the colour-

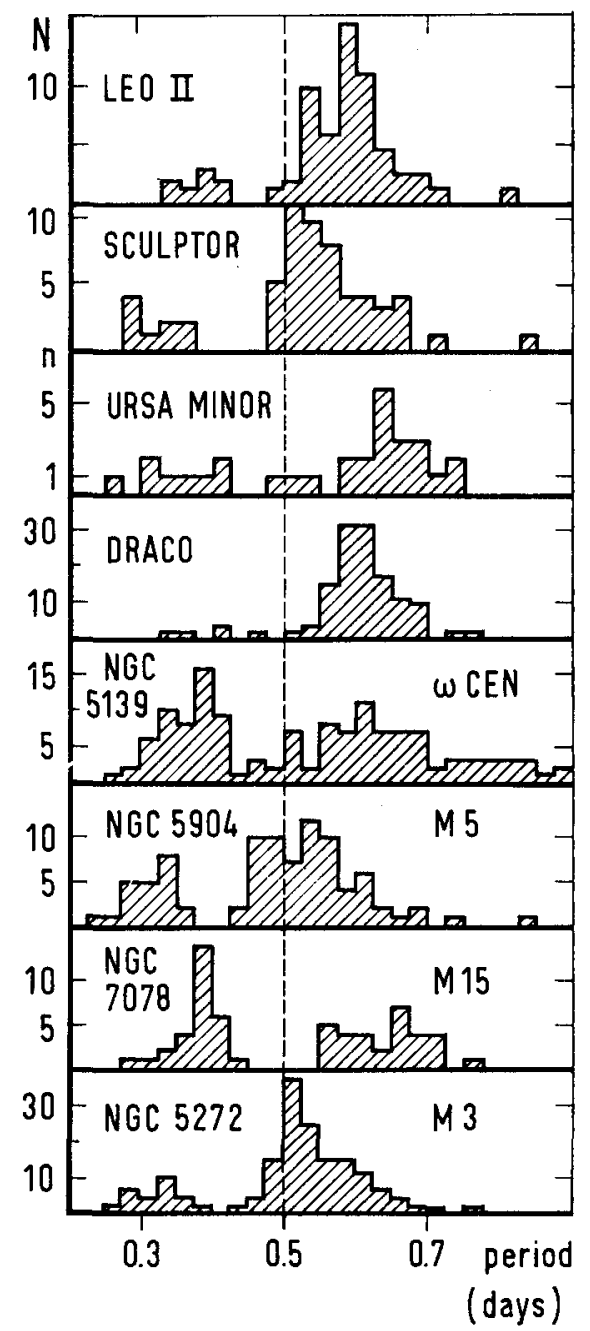

Fig. 1. Period-frequency diagrams for RR Lyrae stars in various dwarf spheroidals and globular clusters. 
magnitude diagram; about where the main-sequence would have been if it continued upwards. It is not known if the star is irregular or eclipsing, or even if it belongs to the Draco system.

In the Leo II system, which has been investigated by Henrietta Swope (Swope, 1967), the total number of variable stars expected to be present was estimated by Baade at close to 250.196 were discovered, and 87 of these have been measured. The percentage of $c$-type variables in this sample is only 9 percent. The colour-magnitude diagram, which is not calibrated in colour, shows a strong concentration of stars towards the red of the horizontal branch.

In the Leo II system the short period Cepheids with period less than one day are also brighter than the RR Lyrae stars in the same spheroidal.

For the Draco, Ursa Minor, Sculptor and the Leo II systems the period-frequency diagrams are brought together in Figure 1. In the same figure are also shown the period-frequency diagrams for the Galactic globular clusters NGC 5139, NGC 5272, NGC 7078 and NGC 5904. The data for the Leo II system are taken from unpublished data by Henrietta Swope.

Selection effects are at work in the diagram for the Sculptor system, where periods have been determined for a relatively small selection of variable stars. There is a possibility that those with larger amplitudes have been chosen preferentially. In the Ursa Minor system the number of variable stars in the central region is small. The period-frequency diagram for Sculptor is very similar to that for NGC 5272. In both diagrams the distribution is smooth and does not show double maxima. The distribution of the periods in the Draco system is comparable, but is shifted towards longer periods.

The distribution of the periods in the Draco system and the Leo II dwarf galaxy strongly disagrees with the concept of the two period groups for the Galactic globular clusters (van Agt and Oosterhoff, 1959). For Galactic globular clusters with sufficient numbers of variable stars, the mean period for the type-a variables in each cluster is for group I (the long period group), $P=0.647 \pm 0.015$, and for group II (the short short period group) $P=0.549 \pm 0.010$. The number of variable stars involved in the mean period of the $\mathrm{RR}_{a}$ variables in the dwarf spheroidal galaxies is sufficiently large to make the deviation from the mean periods of the two globular cluster groups meaningful.

For the dwarf spheroidal galaxies the mean periods are as shown in Table VI:

TABLE VI

Mean periods of RR Lyrae stars in spheroidal galaxies

\begin{tabular}{lll}
\hline Name & $P_{\text {mean }}$ & $N_{c} / N_{\text {total }}$ \\
\hline Sculptor & 0.565 & $0.14:$ \\
Draco & 0.611 & 0.04 \\
Ursa Minor & 0.636 & 0.35 \\
Leo II & 0.592 & 0.09 \\
\hline
\end{tabular}


The mean period of the $\mathbf{R R}_{a}$ variable stars in dwarf spheroidal galaxies clearly does not follow the pattern of the two period groups for Galactic globular clusters but has values inbetween those.

The period-amplitude relations for the dwarf galaxies are given in Figure 2, together with the period-amplitude relations for NGC 5139 and NGC 5272. The general pattern of decreasing amplitude with increasing period as observed for Galactic RR Lyrae stars is retained. Although one should be aware of the serious errors which

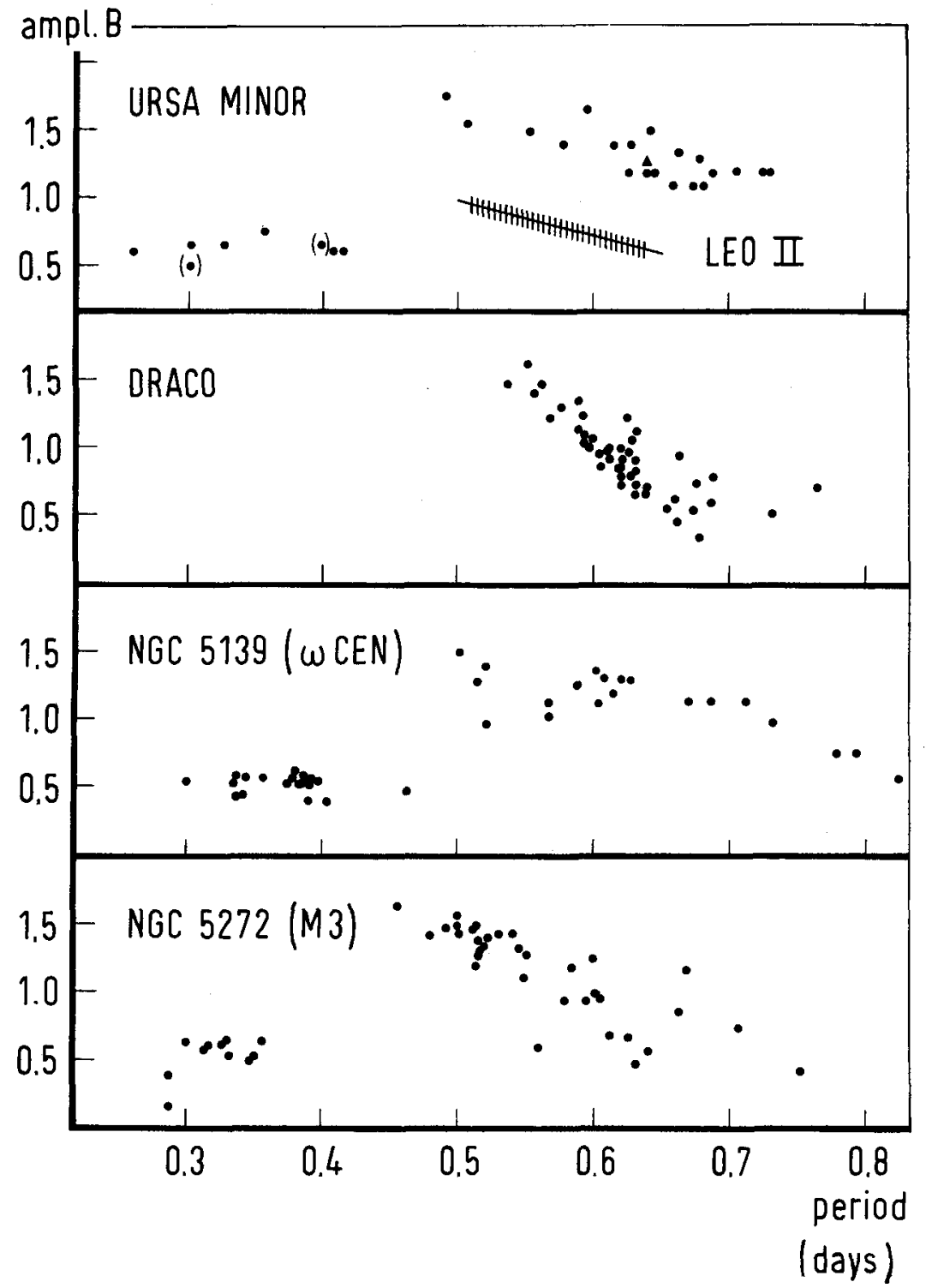

Fig. 2. Period-amplitude diagrams for RR Lyrae stars in various dwarf spheroidals and globular clusters. 
can be introduced by incorrect magnitude scales, it seems evident from the figure that the slope of the period-amplitude relation varies distinctly from cluster to cluster. The slopes for the RR Lyrae stars in NGC 5139 and Ursa Minor system are comparable and, although the amplitudes reached in the Leo II system are considerably smaller, the slope for this dwarf spheroidal does not deviate much from the former two. The maximum amplitude in the Ursa Minor system is approximately 0.5 mag. larger than observed for the Leo II galaxy.

The Draco system is exceptional in the sense that the largest amplitudes are reached at longer periods than in either NGC 5272 or Ursa Minor. The slope of the periodamplitude relation is steeper and the relation is shifted as a whole towards longer periods.

If the conditions in the outer regions of the variable stars on the horizontal branch determine the amplitude of the brightness variations at a certain period then certainly a common factor must be at work for the variable stars in each cluster.

For the Sculptor dwarf spheroidal galaxy the period-amplitude relation is not yet available. However preliminary results for a number of variable stars, which are calibrated with the photographically transferred scale in the Small Cloud globular cluster Kron 3 (Walker, 1970) and NGC 121 (Tifft, 1963), indicate that the variable stars with periods near 0.5 day have amplitudes near 1.5 to $1.7 \mathrm{mag}$. These magnitudes are in the $B_{j}$ system, derived from plates with IIIaJ emulsion exposed behind a GG13 filter.

The period-luminosity relation for the variable stars in the Draco dwarf galaxy is given by Swope (1961), and for the Ursa Minor system by van Agt (1967). For the Leo II system the magnitude scale is still provisional. The average apparent brightness of the RR Lyrae stars in five dwarf spheroidals is given below (Table VII).

TABLE VII

Average apparent magnitudes of RR Lyrae stars

\begin{tabular}{lc}
\hline Name & $B_{\mathbf{R R}}$ \\
\hline Fornax & $21.50:$ \\
Ursa Minor & 20.32 \\
Draco & 20.48 \\
Leo I & - \\
Leo II & 21.90 \\
Sculptor & $20.60:$ \\
\hline
\end{tabular}

Henrietta Swope remarks in her discussion of the period-luminosity relation of the Draco system that there might exist a continuous relation between the stars with period longer than 0.7 and the short period Cepheids in the dwarf spheroidal galaxies. The short period Cepheids in the dwarf spheroidal galaxies all have periods less than 2.7 (V 6 in the Ursa Minor dwarf galaxy), and are systematically brighter by about one magnitude relative to the Cepheids of the same period in globular clusters. 
It is remarkable that the less luminous short period Cepheids found in globular clusters have not been found in the dwarf spheroidals. In addition to the bright short period Cepheids with period close to one day, several dwarf spheroidal galaxies do contain variable stars with periods in the vicinity of 0.5 , but with luminosities 0.4 to 0.8 mag. brighter than the RR Lyrae stars in the same galaxy (Baade and Swope, 1961; Swope, 1968; van Agt, 1967).

The available data are plotted in Figure 3 which suggests that a secondary periodluminosity relation may exist connecting the bright RR Lyrae with the short period Cepheids. This assumes that the RR Lyrae stars proper all have the same luminosity.

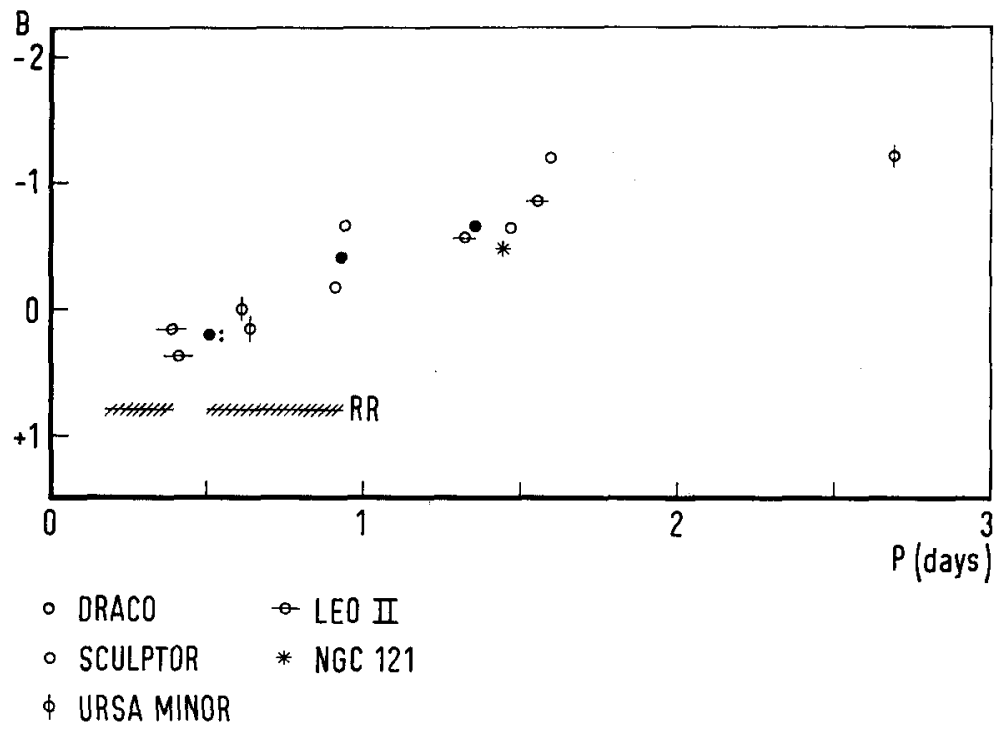

Fig. 3. Composite period-luminosity relation for variables in several systems.

It is unlikely that the bright RR Lyraes and bright Cepheids are foreground stars. The density of stars of this type at the distance of the dwarf spheroidals is too small to explain the observed frequency.

The period-amplitude relation for the small Magellanic Cloud variables indicates maximum amplitudes for periods near one day, and the period-frequency relation for this galaxy also shows a maximum at approximately the same short period. Probably large amplitudes and frequent occurrence of variables continues towards periods considerably shorter than one day for the SMC. If the period-luminosity relation in the SMC is comparable to that suggested for the dwarf spheroidal galaxies, it is likely that the large numbers of short period variable stars found by Dessy (1959) and by Wesselink (1965), at an apparent magnitude too bright for RR Lyrae stars are similar to those in dwarf galaxies. The short period Cepheid No. 6-31 in the field of NGC 121 is located $8^{\prime}$ from the centre of the cluster and has a period of 1.4. If its absolute magnitude is taken to be that given by Figure 3, the distance modulus of NGC 121 is in agreement with the modulus of the SMC. 
Although there are a number of observations (van den Bergh, 1972b) which indicate that the dwarf spheroidal galaxies may have a population similar to part of the Small Magellanic Cloud, it is still essential that more of an effort be made to determine periods and, particularly, to improve the calibration of magnitudes for variable stars in the spheroidals. Until this is done the possibilities offered by these stars in conjunction with quantitative theories of stellar evolution and stellar pulsation cannot be realized.

Note added in proof. Variable star V 80 listed tentatively as a long period variable in Table $\mathrm{V}$ and located in the Ursa Minor galaxy has been investigated by P. N. Kholopov (1971, Astron. Tsirk, No 601, 1) who suggests that this variable is an eclipsing Algol system with one component probably an RR Lyrae variable.

\section{References}

Agt, S. van and Oosterhoff, P.: 1959, Ann. Sterrew. Leiden 21, 253.

Agt, S. van: 1967, Bull. Astron. Inst. Neth. 19, 275.

Baade, W. and Hubble, E.: 1939, Publ. Astron. Soc. Pacific 51, 40.

Baade, W. and Swope, H.: 1961, Astron. J. 66, 300.

Bergh, S. van den: 1968, J. Roy. Astron. Soc. Can. 62, 1.

Bergh, S. van den: 1972a, Publ. David Dunlap Obs., No. 312.

Bergh, S. van den: 1972b, Astrophys. J. Letters 171, L31.

Demers, S.: 1972, J. Roy. Astron. Soc. Can. 66, 215.

Dessy, J.: 1959, Publ. Astron. Soc. Pacific 71, 435.

Gent, H. van: 1933, Bull. Astron. Inst. Neth. 7, 21.

Hodge, P.: 1961, Astron. J. 66, 384.

Hodge, P.: 1964, Astron. J. 69, 853.

Hodge, P.: 1965, Astrophys. J. 142, 1390.

Hodge, P. and Michie, R.: 1969, Astron. J. 74, 587.

Holmberg, E.: 1969, Arkiv Astron. 5, 305.

Iben, I.: 1971, Astron. J. 83, 697.

Marchant, J. and Millikan, E.: 1965, J. Opt. Soc. Am. 55, 907.

Reaves, G.: 1956, Astron. J. 61, 69.

Sandage, A.: 1954, Astron. J. 59, 162.

Shapley, H.: 1938a, Harvard Bull. No. 908.

Shapley, H.: 1938b, Nature 142, 715.

Swope, H.: 1967, Publ. Astron. Soc. Pacific 79, 439.

Swope, H.: 1968, Astron. J. Suppl. 73, 204.

Thackeray, A.: 1950a, Observatory 70, 144.

Thackeray, A.: 1950b, unpublished.

Tifft, W.: 1963, Monthly Notices Roy. Astron. Soc. 126, 209.

Walker, M.: 1970, Astrophys. J. 161, 835.

Wesselink, A.: 1965, Monthly Notices Roy. Astron. Soc. 130, 443.

Wilson, A.: 1955, Pub. Astron. Soc. Pacific 67, 27.

\section{DISCUSSION}

Graham: The population II Cepheid you mentioned as belonging to NGC 121 is far from the cluster and is probably not associated with it. It is more likely to be a field member of the Small Magellanic Cloud population.

van Agt: Variable star 6-31 in the field of NGC 121 has a period at 1.4300 and varies in luminosity 
between 17.9 and 18.8 in $B$ and between 18.25 and 17.65 in $V$. The star is located at a distance of $8^{\prime}$ from the center of NGC 121, for which the radius is somewhat smaller. The star might be a member of the Small Magellanic Cloud field.

Graham: It is most likely that immediate progress on obtaining good faint magnitude sequences near dwarf systems can be made using the Racine prism technique.

van Agt: The Racine wedge is extremely promising and we hope to obtain observations using this technique soon.

Schwarzschild: What is the frequency of occurrence of long period variables and the height of the giant branch?

van Agt: The only dwarf spheroidal which is known to contain a long period variable is the Sculptor system. The calibration of the giant branch points to possibly brighter stars at the tip of the branch relative to the RR Lyrae stars than generally found in metal poor globular clusters. However the calibration is still uncertain. 